\title{
Adaptive MIMO-VLC System for High Data Rate Communications
}

\author{
Fangxiao Dong, Ravinder Singh, and Dominic O'Brien, Member IEEE \\ Department of Engineering Science, University of Oxford, Parks Road, Oxford, OX1 3PJ, UK \\ Email: fangxiao.dong@mansfield.ox.ac.uk, \{ravinder.singh,dominic.obrien\}@eng.ox.ac.uk
}

\begin{abstract}
In this paper, we describe a high-speed indoor MIMO-VLC system that adapts the number of MIMO channels and data rate according to channel conditions at user locations. At each user location across the room, the number of MIMO transmission channels is varied (by grouping different transmitters and sending the same data stream), and the data rate on each channel group is then separately maximised. The system also employs the angle diversity receiver, zero-forcing MIMO equalisation and decision-feedback equalisation to mitigate spatial crosstalk and temporal interference. The performance of the proposed system is evaluated considering a practical indoor MIMO-VLC setup. For the configuration considered a peak overall data rate of $660 \mathrm{Mbps}$ is achieved, and on average the overall data rate is 120 Mbps higher than a conventional spatial multiplexing approach. This shows the potential of this simple allocation scheme, compared with more complex precoding approaches.
\end{abstract}

Keywords-Visible Light Communications (VLC), Multiple-Input Multiple-Output (MIMO), Angle Diversity Receiver, Channel Equalisation, Transmitter Coordination, Data Rate Adaptive Modulation.

\section{INTRODUCTION}

The use of white light-emitting diodes (LEDs) for illumination has led to increasing interest in indoor visible light communications (VLC). Using LEDs, VLC systems can not only modulate the light intensity at high frequencies to transmit data, but also furnish stable indoor illumination [1]. In most indoor scenarios, there are multiple LEDs on the ceiling to illuminate the room, which offers the potential to develop the Multiple-Input Multiple-Output (MIMO) VLC system. MIMO-VLC with multiple transmitters and multiple receivers enables parallel data transmission or spatial multiplexing and de-multiplexing. The potential higher spectral efficiency increases the possibility of high-speed data communication [1], [2].

MIMO technology is already widely implemented in radio-frequency (RF) communication systems where the spectral efficiency is dramatically improved by spatial paths with high diversity [2]. However, the available spatial diversity of MIMO-VLC systems is limited by the intensitymodulation direct-detection (IM/DD) channels. For the IM/DD VLC, the small difference in the channel gains at the MIMO receiver does not give a well-conditioned channel matrix, which makes the decoupling of signals difficult. This defect causes the multi-stream interference (spatial crosstalk) and affects the performance of the high-speed system.

A number of schemes have been proposed to accommodate the VLC system with multiple transmitters and multiple receivers, such as spatial diversity, spatial multiplexing and spatial modulation [2]. Spatial multiplexing assigns independent data streams to each transmitter and transmits them in parallel. Spectral efficiency can be improved by up to the factor of the number of transmitters $N_{t}$ (see, e.g., [3] - [5]). However, the system performance is suppressed by multi-stream interference raised by poor channel diversity [6]. Basic spatial modulation typically turns on only one transmitter at a time, and the index of the transmitter is decided by the input data bits. The index is estimated at the receiver side, which is used to recapture the transmitted bits. This technique was proposed in [7] and investigated and demonstrated in [8] and [9], as well as further variants such as those that enable light dimming [10]. Spatial modulation improves the spectral efficiency by a factor of $\log _{2}\left(N_{r}\right)$, but the spatially correlated channels also affects the system performance. Ganging is another technique to drive multiple LEDs, in which the same data streams are sent from all transmitters. All the signals are received and processed at the receiver side together, as shown in [11] and [12]. Ganging provides limited spectral efficiency, but it is less restrictive in transmitter and receiver alignment than MIMO as LEDs transmit the same data. In a MIMO system, when the channels are similar and the received signals are hard to decouple at some user locations, enabling ganging can combine similar channels to transmit the same data streams, therefore maintaining a valid communication link.

In this paper, we propose a Channel Condition-based Transmitter Coordination (CCTC) algorithm to deliberately combine ganging and multiplexing schemes to improve the overall data rate of the system. This method makes sure when channels are well-conditioned, all channels can transmit different data sequences to achieve a high overall data rate as in spatial multiplexing. However, when some of the channels have strong spatial crosstalk between them at some locations in the receiver plane, the system will switch to a full or partially ganged scheme based on a given Bit Error Rate (BER) threshold to improve the overall performance. A data rate adaptive modulation is also introduced to optimise the data rate of each separate channel. Both the transmitter patterns and the data rate per data stream are carefully decided for each user location in the room with a required BER. Precoding has also been proposed to mitigate the spatial crosstalk in MIMO-VLC systems (see, e.g., [13] - [15]). The approach taken in this paper uses the same modulation scheme and the same optical power for each transmitter, which maintains a constant illumination level from each source while making efficient use of the available source dynamic range.

The investigated MIMO system also employs an angle diversity receiver, zero-forcing MIMO equalisation and decision feedback equalisation to decrease the spatial crosstalk and temporal crosstalk. The performance of the proposed data rate adaptive CCTC MIMO-VLC system is studied by modelling a practical indoor VLC scenario. The achievable overall data rate (summed for independent data 


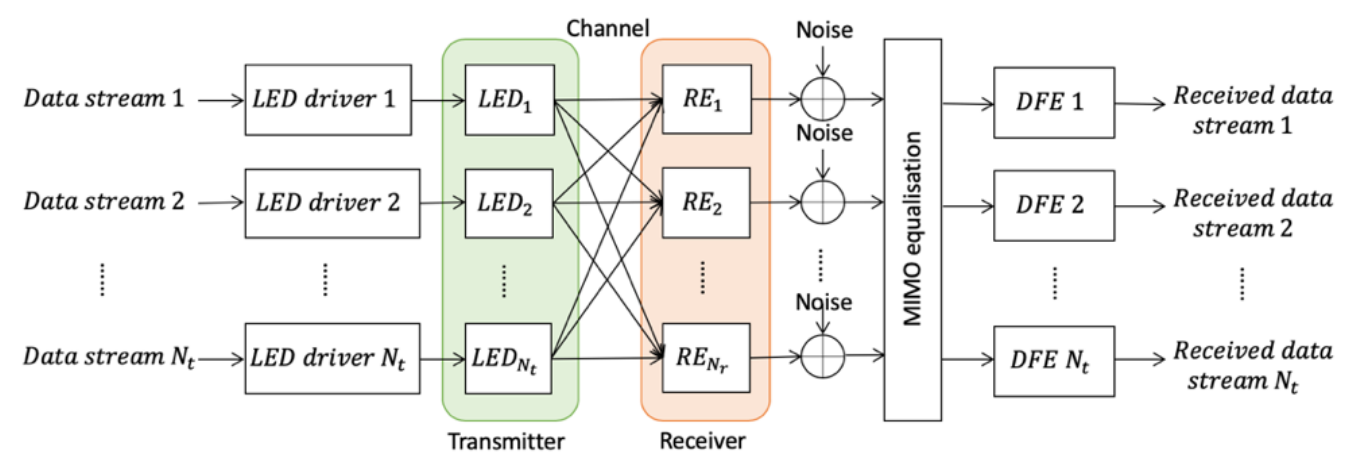

Fig. 2. Schematic of the indoor MIMO-VLC system.

streams) across the room is evaluated and compared to the conventional spatial multiplexing and CCTC based data rate non-adaptive systems.

The rest of the paper is organised as follows. Section II describes the system model and the angle diversity receiver design. Section III introduces the data rate adaptive modulation technique and the CCTC algorithm. Section IV compares the performance of the new system with that of the non-adaptive MIMO-VLC systems. Finally, the conclusions and future works are discussed in Section V.

\section{System Model}

Fig. 1 shows a schematic of typical MIMO-VLC system [16]. $N_{t}$ LEDs are installed on the ceiling, illuminating the room as well as transmitting information to the receiver. A receiver with $N_{r}$ receiver elements (RE) is implemented on the receiver plane. Each RE receives the light from multiple LEDs, but with different light intensity. The channels between $N_{t}$ transmitters and $N_{r}$ REs construct an $N_{r} \times N_{t}$ channel matrix, $\boldsymbol{H}$. There are assumptions that $N_{t}$ transmitter data streams are operated by a controller, and that the channel matrix $\boldsymbol{H}$ is perfectly known at the transmitter and the receiver. The parameters of the system are shown in Table I [16]. The schematic of the system model is shown in Fig. 2, which will be explained in detail in the following sections.

TABLE I. SYSTEM PARAMTER

\begin{tabular}{|l|l|}
\hline \multicolumn{1}{|c|}{ Parameters } & \multicolumn{1}{c|}{ Values } \\
\hline Room size (width $\times$ length $\times$ height) & $5 \mathrm{~m} \times 5 \mathrm{~m} \times 3 \mathrm{~m}$ \\
\hline Receiver plane height & $0.85 \mathrm{~m}$ \\
\hline Numbers of LEDs $\left(N_{t}\right)$ & 4 \\
\hline Numbers of REs $\left(N_{r}\right)$ & 7 \\
\hline Modulation power per LED $\left(P_{L E D}\right)$ & $1 \mathrm{~W}$ \\
\hline LED pitch & $2.5 \mathrm{~m}$ \\
\hline $\begin{array}{l}\text { Lambertian order }(m) \text { (transmitter semi-angle } \\
\left.\left(\theta_{1 / 2}\right)\right)\end{array}$ & $1\left(60^{\circ}\right)$ \\
\hline Collection area of each RE $\left(\mathrm{A}_{\mathrm{j}}\right)$ & $100 \mathrm{~mm}^{2}$ \\
\hline Half-angle field of view of receiver $\left(\beta_{\mathrm{c}}\right)$ & $33^{\circ}$ \\
\hline Concentrator refractive index $\left(n_{c}\right)$ & 1.5 \\
\hline LED and Receiver bandwidth & $8.4 \mathrm{MHz}$ \\
\hline Detector responsivity $(\rho)$ & $0.4 \mathrm{~A} / \mathrm{W}[16]$ \\
\hline Pre-amplifier noise current density $\left(i_{\text {amp }}\right)$ & $5 \mathrm{pA} / \mathrm{Hz}^{1 / 2}[16]$ \\
\hline Ambient light photocurrent density $\left(\gamma_{\text {ambient }}\right)$ & $10.93 \mathrm{~A} /\left(\mathrm{m}^{2} \cdot \mathrm{Sr}\right)[17]$ \\
\hline
\end{tabular}

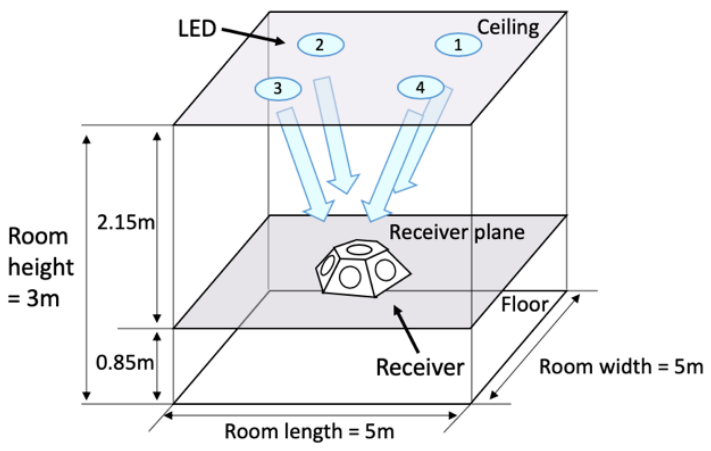

Fig. 1. Configuration of typical indoor MIMO-VLC system.

\section{A. Transmitter}

The LEDs are modelled as Lambertian sources. The Lambertian source order $m$ can be derived from the LED semi-angle $\theta_{1 / 2}$ at half power as [18]

$$
m=\frac{\ln \left(\frac{1}{2}\right)}{\ln \left(\cos \left(\theta_{1 / 2}\right)\right)},
$$

In this work, 4 LEDs are arranged as a $2 \times 2$ array with 2.5 $m$ pitches. A Non-Return to Zero On-Off Keying (NRZ-OOK) modulation scheme is used to transfer data. The binary data streams are converted to electrical signals which modulate the intensity of the LEDs. Each electrical signal is convolved with the impulse response of LED when it passes through the LED [19]

$$
h_{L E D}(t)=e^{-\omega_{c} t},
$$

where $\omega_{c}$ is the $3 \mathrm{~dB}$ cut-off bandwidth of the LED in angular frequency. After the LED, the vector $\boldsymbol{D}=\left[d_{1}, \ldots d_{i} \ldots, d_{N_{t}}\right]^{T}$ is obtained at each time instance. $d_{i}$ represents the convolution of the $i$ th data stream and the impulse response of the $i$ th LED. $T$ presents the transpose of the matrix.

\section{B. Wireless Channel between Transmitter and Receiver}

$\boldsymbol{H}$ is the $N_{r} \times N_{t}$ channel matrix

$$
\boldsymbol{H}=\left[\begin{array}{ccccc}
h_{11} & \ldots & h_{1 i} & \ldots & h_{1 N_{t}} \\
\vdots & \ddots & \vdots & & \vdots \\
h_{j 1} & \ldots & h_{j i} & \ldots & h_{11} \\
\vdots & & \vdots & \ddots & \vdots \\
h_{N_{r} 1} & \ldots & h_{N_{r} i} & \ldots & h_{N_{r} N_{t}}
\end{array}\right]
$$

where $h_{j i}$ shows the channel gain between $i$ th LED and $j$ th RE. $h_{j i}$ is given as 


$$
h_{j i}=\left\{\begin{array}{cc}
\frac{(m+1) A_{j}}{2 \pi d_{j i}{ }^{2}} \cos ^{m}\left(\alpha_{j i}\right) \cos \left(\beta_{j i}\right) & 0 \leq \beta_{j i} \leq \beta_{c} \\
0 & \beta_{j i}>\beta_{c}
\end{array},\right.
$$

where $A_{j}$ is the collection area of the $j$ th RE, $d_{j i}$ is the distance between the $i$ th LED and the $j$ th RE, $\alpha_{j i}$ is the irradiance angle at the $i$ th LED with respect to the $j$ th RE, $\beta_{j i}$ is the incident angle at the $j$ th RE with respect to the $i$ th LED, $\beta_{c}$ is the receiver half-angle Field of View (FoV).

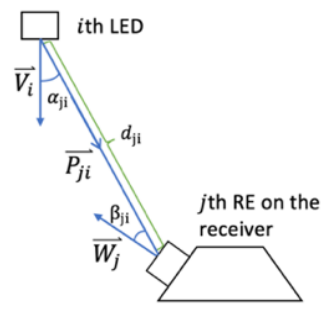

Fig. 3. Relative geometry between a LED and RE pair.

In order to obtain $\alpha_{j i}$ and $d_{j i}$, three vectors between the $i$ th LED and the $j$ th RE are defined in Fig. 3 [20]. $\vec{V}_{l}$ is the normal vector of the $i$ th LED, pointing vertically downwards. $\vec{W}_{J}$ is the normal vector of the $j$ th RE, $\overrightarrow{P_{l l}}$ is the vector pointing from the $i$ th LED to the $j$ th RE. $\alpha_{j i}$ and $\beta_{j i}$ can be derived as

$$
\begin{aligned}
& \alpha_{j i}=\cos ^{-1}\left(\frac{\overrightarrow{V_{l}} \cdot \overrightarrow{P_{l}}}{\left\|\overrightarrow{V_{l}}\right\|\left\|\overrightarrow{P_{\jmath}}\right\|}\right), \\
& \beta_{j i}=\cos ^{-1}\left(\frac{\overrightarrow{P_{l}} \cdot \overrightarrow{W_{J}}}{\left\|\overrightarrow{P_{\jmath l}}\right\|\left\|\overline{W_{J}}\right\|}\right),
\end{aligned}
$$

\section{Receiver}

The signal received by REs at a time instance is given by

$$
\boldsymbol{R}=\rho P_{L E D}(\boldsymbol{H} \bullet \boldsymbol{D})+\boldsymbol{i}_{N},
$$

where $\boldsymbol{R}=\left[r_{1}, \ldots r_{j} \ldots, r_{N_{r}}\right]^{T}, \rho$ is detector responsivity, $\boldsymbol{i}_{N}=$ $\left[i_{n, 1}, \ldots i_{n, j} \ldots, i_{n, N_{r}}\right]^{T}$ is the noise current vector of the preamplifier. $i_{n, j}$ for each RE is an independent identically distributed (i.i.d.) AWGN random variable [20].

$P_{L E D}$ is the modulation power per LED as given in Table I. When transmitting data streams using OOK, 0 and 1 are transmitted by decreasing and increasing the intensity of the LED light wave to two different levels. These two levels of light intensity are achieved by controlling the LED modulation power.

The noise received by an RE consists of optical signal noise, ambient noise and amplifier noise. The signal noise and ambient noise are the shot noise from the desired signal and the detection, while the amplifier noise involves all the thermal and shot noise relevant to the pre-amplifier circuit [21]. The noise variance is calculated from the mean square of the noise current

$$
\overline{l_{n, J}^{2}}=2 e\left(i_{\text {signal }}^{j}+i_{\text {ambient }}^{j}\right) B+i_{a m p}^{2} B,
$$

where $e$ is the elementary positive charge, $i_{a m p}$ is the preamplifier noise current density and B is the bandwidth of the receiver, $i_{\text {signal }}^{j}$ is the average received current density for the $j$ th RE,

$$
i_{\text {signal }}^{j}=\rho P_{L E D} \sum_{i=1}^{N_{t}} h_{j i} d_{i}
$$

$i_{\text {ambient }}^{j}$ represents the received current density from the ambient light for the $j$ th $\mathrm{RE}$

$$
i_{\text {ambient }}^{j}=\gamma_{\text {ambient }} A_{j} 2 \pi\left(1-\cos \left(\beta_{c}\right)\right),
$$

where $\gamma_{\text {ambient }}$ is the ambient light photocurrent per square metre per steradian, $2 \pi\left(1-\cos \left(\beta_{c}\right)\right)$ is the solid angle of a cone corresponding to the cross-section with the angle $2 \beta_{c}$.

A linear zero-forcing equalizer is applied as the demultiplexing technique to decouple the channel matrix. The estimated vector $\widehat{\boldsymbol{D}}$ of the transmitted signals can be calculated by

$$
\widehat{D}=H_{Z F} \cdot R,
$$

where

$$
\boldsymbol{H}_{Z \boldsymbol{F}} \stackrel{\text { def }}{=}\left(\boldsymbol{H}^{*} \boldsymbol{H}\right)^{-1} \boldsymbol{H}^{*},
$$

denotes the Moore-Penrose inverse of $\boldsymbol{H}$, which gives the best approximate solution for non-squared matrices and non-full rank square matrices [20]. $\boldsymbol{H}^{*}$ is the conjugated transpose of $\boldsymbol{H}$. (14) helps to find the estimated $\widehat{\boldsymbol{D}}$ such that the Euclidean norm $\left\|\rho P_{L E D}(\boldsymbol{H} \bullet \boldsymbol{D})+\boldsymbol{i}_{N}-\boldsymbol{R}\right\|$ is minimised [22].

Decision-feedback equalisation (DFE) is employed to overcome inter-symbol interference after the MIMO demultiplexing [23]. Four DFEs are employed in parallel to process the data separately for four channels, which may be operating at different data rates. During the operation, a training mode is used at first to optimise the weighting coefficients of DFEs. The real data streams are processed by the DFEs after the DFEs are well-trained.

\section{Considered Angular Diversity Receiver Design}

Fig. 4 shows a seven-channel angle diversity receiver (ADR). The top view of the ADR shows 6-fold rotational symmetry, and the angle between adjacent REs is $60^{\circ}$. From the front view, it can be seen that the pointing direction of RE 1 to 6 has an upward angle $30^{\circ}$ relative to the horizontal receiver plane. Only RE 7 points vertically upwards.

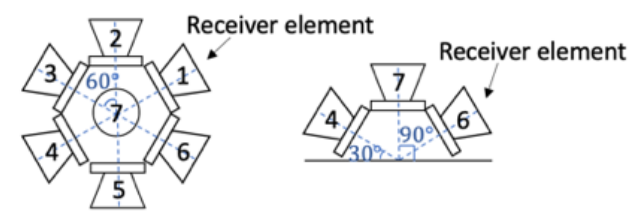

Fig. 4. Geometry of the receiver: top view (left) and front view (right).

Each RE is made up of a non-imaging concentrator to gather the radiation, a photodetector to convert optical power to photocurrent, and a pre-amplifier to amplify the signal. The receiver collection area is given by

$$
A_{j}=\frac{n_{c}^{2}}{\sin ^{2}\left(\beta_{c}\right)} A_{d e t}
$$

where $n_{c}$ is the ideal optical concentrator refractive index, $A_{\text {det }}$ is the detection area of the photodetector.

The collection area $A_{j}$ of each RE is $100 \mathrm{~mm}^{2}$. The halfangle field of view $\left(\beta_{c}\right)$ of each $R E$ is $33^{\circ}$, which limits the 
number of transmitted signals each receiver can receive, thus reducing the spatial crosstalk.

Because of the small distances between each RE compared to the distances between LEDs and REs, it is assumed that the location of the REs is at a same point. Choosing the number of receivers greater than the number of transmitters helps the receiver to decouple the signals easier.

\section{Data Rate Adaptive Modulation With TRANSMITTER COORDINATION}

\section{A. Data Rate Adaptive Modulation}

In an indoor MIMO-VLC system, different transmitters typically have the same data rate, such as the model in [16]. However, with multiple channels in the system, some channels are affected by higher interference in some specific receiver locations. These channels can have much higher BER than other high-quality channels. With the same BER requirement, a good channel is expected to achieve a higher data rate than the poor channel. In this circumstance, having adaptive data rates depending on the channel condition can make sure each channel reaches its optimised data rate.

Here a data rate adaptive modulation technique is proposed. The basic idea behind the data rate adaptive modulation is to realise the time-alignment of waveforms by adjusting the up-sampling factor $U_{f}$ of each channel based on the desired data rate. Fig. 7 is a simple schematic representing the mechanism behind the data rate adaptive technique. Two waveforms represent signals from two sources within the packet duration $t_{\text {packet }}$. The sample period $T_{\text {sample }}$ is identical in all channels, and the total number of samples $N_{\text {sample }}$ also matches. The data rate of waveform 2 is twice as fast as the waveform 1 . In this circumstance, the up-sampling factor of the high data rate signal is half of the up-sampling factor of the low data rate signal. Hence the waveforms with different data rates keep being time-aligned throughout the packet time, and the overall time spent on transmitting the packets with different length is the same for all channels.

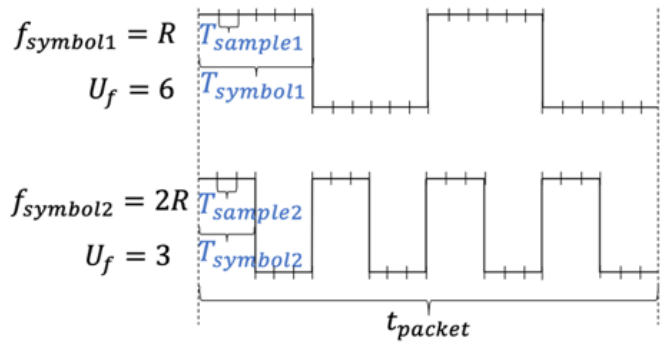

Fig. 7. Schematic of the data rate adaptive technique.

In the system settings, the up-sampling factor range should be decided appropriately at first. Then a reasonable number of symbols per packet $N_{\text {symbol }}$ and data rate $f_{\text {symbol }}$ range can be derived from the up-sampling factor.

The number of symbols in each packet is calculated as

$$
N_{\text {symbol }}=\frac{N_{\text {sample }}}{U_{f}}=\frac{t_{\text {packet }}}{T_{\text {sample }} \times U_{f}},
$$

where $N_{\text {sample }}$ is the number of samples in a packet, and $T_{\text {sample }}$ is the sample period. The data rates is

$$
f_{\text {symbol }}=\frac{1}{T_{\text {symbol }}}=\frac{1}{T_{\text {sample }} \times U_{f}},
$$

\section{B. Transmitter Coordination Algorithm}

Here, a MIMO scheme combining the ganging and spatial multiplexing is introduced to improve the overall data rates over the receiver plane. An algorithm is introduced to coordinate patterns of LEDs. At each user location, the BER for each transmission is monitored. The proposed algorithm makes a decision on whether to use a full ganging, spatial multiplexing or the combination of spatial multiplexing and ganging, depending on if the average BER is greater or smaller than $1 \times 10^{-3}$. Then it determines how high the data rates should be set for the selected pattern with the BER requirement.

Fig. 6 shows the CCTC patterns with different grouping of channels. If we have four channels transmitting different data streams, there is one possible LED pattern, pattern 1. For pattern 2, there are six possible patterns, as three different data streams transmitted. For pattern 3, there are seven patterns with two independent channels. For pattern 4 , there is only one pattern and all channels transmit the same data stream.

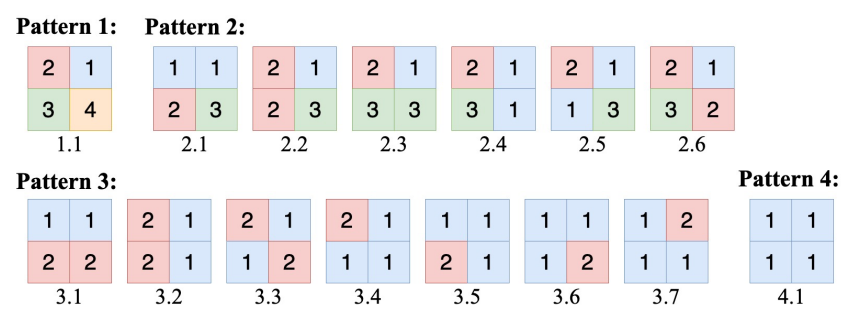

Fig. 6. Channel condition based transmitter coordination patterns. $\mathrm{z}$

The transmitter operation is summarised in Algorithm 1.

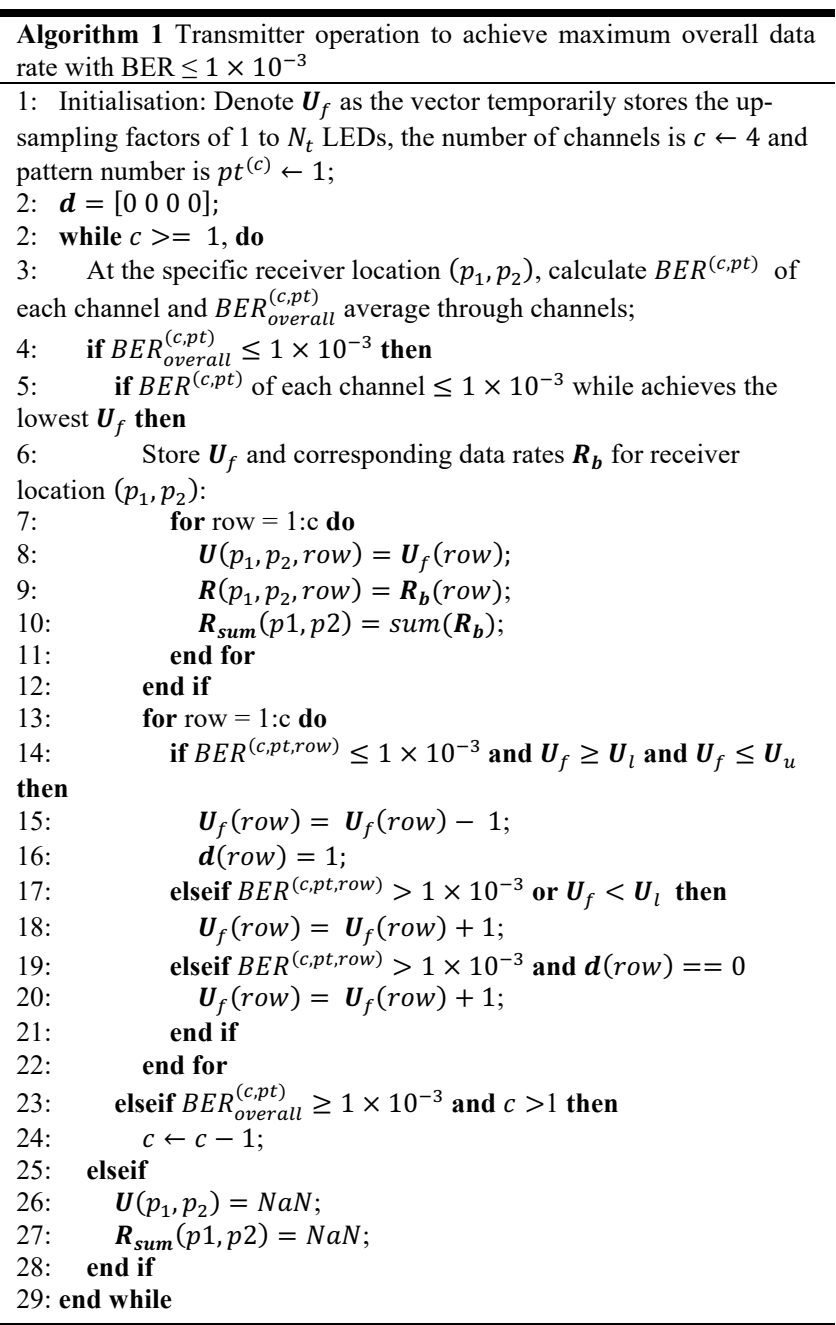




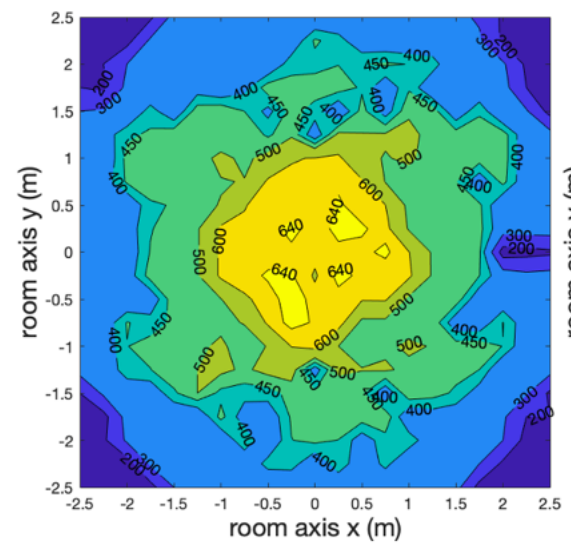

(a)

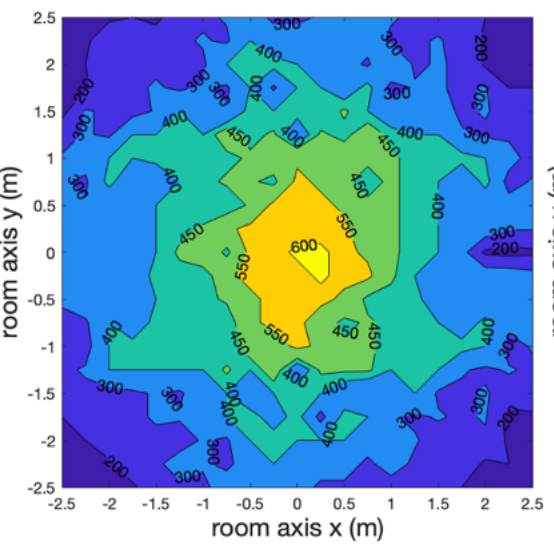

(b)

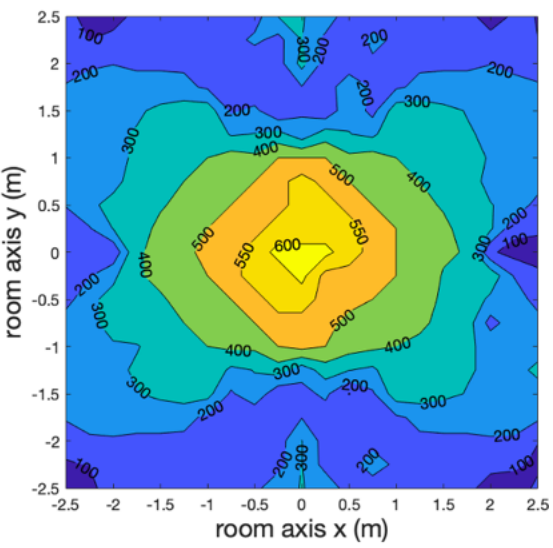

(c)

Fig. 8. Overall data rate (Mbps) distribution for, (a) case A: the proposed system with adaptive data rate and Algorithm 1, (b) case B: the system with non-adaptive data rate and Algorithm 1, (c) case C: the conventional system with non-adaptive data rate and spatial multiplexing.

In Algorithm 1, $\boldsymbol{U}_{f}$ is the vector temporarily stores the values of the up-sampling factor with the lower and upper bound $\boldsymbol{U}_{l}$ and $\boldsymbol{U}_{u} . \boldsymbol{U}$ is the matrix saves the final values of the up-sampling factors at each receiver position across the receiver plane. $\boldsymbol{R}$ is the matrix saves the final data rates for all channels, $\boldsymbol{R}_{\text {sum }}$ is the matrix saves the final overall data rate at each user location across the receiver plane.

The BER is acquired by comparing the estimated bits and the transmitted bits. The reason for using BER $\leq 1 \times 10^{-3}$ is because the value is below the forward error correction (FEC) limit $3.8 \times 10^{-3}[24]$.

\section{Simulation And Results}

In this section, we analyse the overall data rate performance of the MIMO techniques introduced in Section III, considering several setup scenarios.

\section{A. System settings}

At the transmitter, up-sampling factor ranging from 6 to 20 is used, which results in an achievable data rate from 100 Mbps to $333 \mathrm{Mbps}$ for each channel with specific increments. This range provides a sufficient data rate range to choose from when applying Algorithm 1. The transmission time of each packet $t_{\text {packet }}$ is set to $0.1 \mathrm{~ms}$ and the sample duration $T_{\text {sample }}$ is set to $0.5 \mathrm{~ns}$. Hence there are 200 thousand samples per packet using (15). In total, 50 packets are transmitted, over which the obtained values of BER are averaged. Perfect knowledge of the channel matrix is assumed.

At the receiver side, the DFE uses 24 feedforward and 19 feedback filter taps. The training length is 400 thousand samples for each user location. The LMS algorithm is applied to estimate the tap weights. The training mode is only used in the first iteration to train the DFE.

\section{B. Performance Comparison}

Fig. 8 shows the distributions of the overall data rate over the receiver plane with and without applying Algorithm 1 and data rate adaptive modulation, respectively. We refer to these as case A, case B and case C. Fig. 9(a) and Fig. 9(b) provide the number of independent communication channels for Fig. 8(a) and Fig. 8(b), respectively. For Fig. 8(c), the number of channels remains four across the room. From these figures it can be seen that the overall data rates increase progressively from the edge of the room to its centre. The figures are expected to be perfect symmetric around the $\mathrm{x}$-axis and $\mathrm{y}$-axis given the system geometry. The figures show asymmetry around both the $\mathrm{x}$-axis and $\mathrm{y}$-axis due to the limited resolution of the simulation and a limited number of transmitted packets.

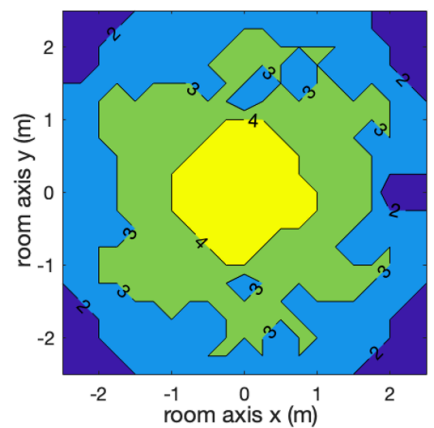

(a)

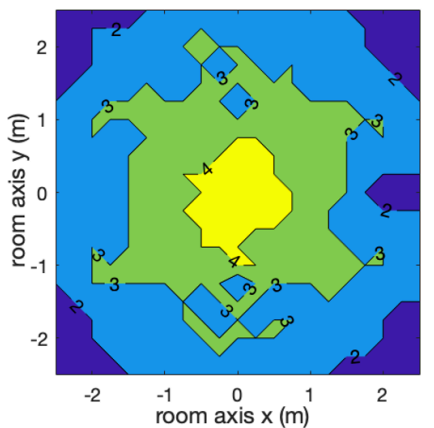

(b)
Fig. 9. Number of channels distribution for, (a) case A: the proposed system with adaptive data rate and Algorithm 1, (b) case B: the system with non-adaptive data rate and Algorithm 1.

Firstly, the performance of partial ganging and spatial multiplexing is compared (case $\mathrm{B}$ and $\mathrm{C}$ ). In the conventional system that uses spatial multiplexing, the overall data rate decreases from the middle to the edge. This trend results from the increased condition number of the channel matrix and the decreased SINR of the system. With the employment of Algorithm 1, the impact of the ill-conditioned channel matrix is mitigated by employing partial ganging. The channels with higher interference and lower SINR are combined to transmit the same data sequence, which significantly improves the data rates of the poor channels. The overall data rate versus receiver distance to the room centre is plotted in Fig. 10 based on the results in Fig. 8. Fig. 10 shows that the system in case $B$ provides a higher overall data rate than that in case $\mathrm{C}$ except for the area in the room centre. The difference in data rate vanishes in the centre of the room because all four channels have good quality here and pure spatial multiplexing is used in both cases in this area. From a holistic perspective, the overall data rate improves around $64 \mathrm{Mbps}$ on average when employing partial ganging in case $\mathrm{B}$ than case $\mathrm{C}$. 


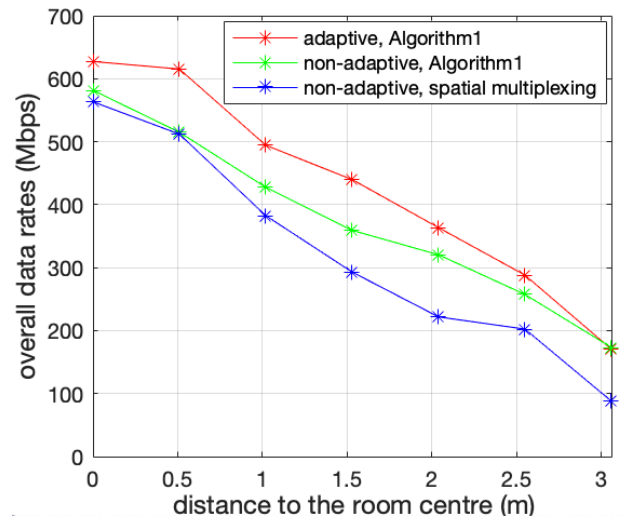

Fig. 10. Overall data rates versus the receiver distance to the room centre.

The data rate performance further increases when data rate adaptive modulation is applied. The highest overall data rate in Fig 8(a) is around $660 \mathrm{Mbps}$. The average overall data rate in case $\mathrm{A}$ is $56 \mathrm{Mbps}$ higher than that in case B. This performance is because of the use of fixed data rate over each channel in case B and even though the poor channels are combined in case B, the channels still have different quality. Channels with higher SINR are capable of transmitting data faster while keeping the BER below the required threshold, which is achieved by the adaptive scheme. The overall data rate improvement also disappears at the four corners of the room because pure ganging is applied here for both case $\mathrm{A}$ and case B. In summary, the employment of CCTC algorithm and data rate adaptive modulation improves the overall data rate by $120 \mathrm{Mbps}$ (on average across the room) in case A when compared to case $\mathrm{C}$.

\section{CONCLUSION}

In this paper, a CCTC algorithm and a data rate adaptive modulation technique are proposed in a typical indoor MIMO-VLC system. The simulation results show that the proposed techniques effectively mitigate spatial crosstalk and temporal interference in the system. Overall, the proposed channel adaptive system outperforms the non-adaptive MIMO-VLC system by achieving higher data rates for every user location across the room. The switching threshold between different number of channels depends on the BER average through all the channels, which makes the switching point not accurate enough. In the future, we intend to investigate the SINR and the structure of the channel matrix to select the optimised transmitter patterns and data rates directly. The combined use of partial spatial modulation, spatial multiplexing and ganging also has the potential to be explored.

\section{REFERENCES}

[1] A. Jovicic, J. Li, and T. Richardson, "Visible light communication: Opportunities, challenges and the path to market," IEEE Commun. Mag., vol. 51, no. 12, pp. 26-32, 2013.

[2] P. H. Pathak, X. Feng, P. Hu, and P. Mohapatra, "Visible Light Communication, Networking, and Sensing: A Survey, Potential and Challenges," IEEE Commun. Surv. Tutorials, vol. 17, no. 4, pp. $2047-$ 2077, 2015.

[3] L. Zeng et al., "High data rate Multiple Input Multiple Output (MIMO) optical wireless communications using white LED lighting," IEEE $J$. Sel. Areas Commun., vol. 27, no. 9, pp. 1654-1662, 2009.

[4] J. Lian, "Communication System Using Spatial Multiplexing," vol. 35, no. 23, pp. 5024-5033, 2017.

[5] K. D. Dambul, D. C. O'Brien, and G. Faulkner, "Indoor optical wireless MIMO system with an imaging receiver," IEEE Photonics Technol. Lett., vol. 23, no. 2, pp. 97-99, 2011.

[6] C. He, T. Q. Wang, and J. Armstrong, "Performance comparison between spatial multiplexing and spatial modulation in indoor MIMO visible light communication systems," 2016 IEEE Int. Conf. Commun. ICC 2016, no. Dp 130101265, pp. 1-6, 2016.

[7] R. Mesleh, H. Haas, C. W. Ahn, and S. Yun, "Spatial modulation - A new low complexity spectral efficiency enhancing technique," First Int. Conf. Commun. Netw. China, ChinaCom '06, 2006.

[8] R. Mesleh, H. Elgala, and H. Haas, "Optical spatial modulation," $J$. Opt. Commun. Netw., vol. 3, no. 3, pp. 234-244, 2011.

[9] E. Poves, W. Popoola, H. Haas, J. Thompson, and D. Cárdenas, "Experimental results on the performance of optical spatial modulation systems," IEEE Veh. Technol. Conf., pp. 1-5, 2012.

[10] T. C. Bui, R. Singh, T. O'Farrell, and M. Biagi, "Performance Evaluation of Generalized Optical Spatial Modulation with Dimming Support," 2018 IEEE Globecom Work. GC Wkshps 2018 - Proc., pp. $1-6,2019$.

[11] H. Le Minh et al., "High-speed visible light communications using multiple-resonant equalization," IEEE Photonics Technol. Lett., vol. 20, no. 14, pp. 1243-1245, 2008.

[12] T. Fath and H. Haas, "Performance comparison of MIMO techniques for optical wireless communications in indoor environments," IEEE Trans. Commun., vol. 61, no. 2, pp. 733-742, 2013.

[13] K. H. Park, Y. C. Ko, and M. S. Alouini, "A novel power and offset allocation method for spatial multiplexing MIMO Systems in optical wireless channels," 2011 IEEE GLOBECOM Work. GC Wkshps 2011, pp. 823-827, 2011.

[14] B. Li, J. Wang, R. Zhang, H. Shen, C. Zhao, and L. Hanzo, "Multiuser MISO transceiver design for indoor downlink visible light communication under per-LED optical power constraints," IEEE Photonics J., vol. 7, no. 4, pp. 1-15, 2015.

[15] H. Sifaou, K. H. Park, A. Kammoun, and M. S. Alouini, "Optimal linear precoding for indoor visible light communication system," IEEE Int. Conf. Commun., 2017.

[16] L. Zeng et al., "High Data Rate Multiple Input Multiple Output ( MIMO ) Optical Wireless Communications Using White LED Lighting," IEEE J. Sel. Areas Commun., vol. 27, no. 9, pp. 1654-1662, 2009.

[17] A. J. C. Moreira, R. T. Valadas, and A. M. De Oliveira Duarte, "Optical interference produced by artificial light," Wirel. Networks, vol. 3, no. 2, pp. 131-140, 1997.

[18] Z. Chen, D. A. Basnayaka, X. Wu, and H. Haas, "Interference mitigation for indoor optical attocell networks using an angle diversity receiver," J. Light. Technol., vol. 36, no. 18, pp. 3866-3881, 2018.

[19] L. Zeng, D. xwO'Brien, H. Le-Minh, K. Lee, D. Jung, and Y. Oh, "Improvement of date rate by using equalization in an indoor visible light communication system," 2008 4th IEEE Int. Conf. Circuits Syst. Commun. ICCSC, no. 0, pp. 678-682, 2008.

[20] A. Nuwanpriya, S. W. Ho, and C. S. Chen, "Indoor MIMO Visible Light Communications: Novel Angle Diversity Receivers for Mobile Users," IEEE J. Sel. Areas Commun., vol. 33, no. 9, pp. 1780-1792, 2015.

[21] D. C. O'Brien and M. Katz, "Optical wireless communications within fourth-generation wireless systems [Invited]," J. Opt. Netw., vol. 4, no. 6, pp. 312-322, 2005.

[22] J. C. A. Barata and M. S. Hussein, "The Moore-Penrose Pseudoinverse: A Tutorial Review of the Theory," Brazilian J. Phys., vol. 42, no. 1-2, pp. 146-165, 2012.

[23] J. M. Cioffi, G. P. Dudevoir, M. V. Eyuboglu, and G. D. Forney, "MMSE Decision-Feedback Equalizers and Coding-Part I: Equalization Results," IEEE Trans. Commun., vol. 43, no. 10, pp. 2582-2594, 1995.

[24] ITU-T Group, "ITU-T Rec. G.975.1 (02/2004) Forward error correction for high bit-rate DWDM submarine systems," Standard, pp. $1-58,2005$. 